\title{
The Covid-19 pandemic: An urgent need to remove silo mentality in science and tackle global health challenges through coordination, cooperation and collaboration
}

\author{
Parvez I. Haris \\ Faculty of Health \& Life Sciences, De Montfort University, Leicester, LE1 9BH, United Kingdom \\ E-mail: pharis@dmu.ac.uk
}

The COVID-19 pandemic, caused by the SARS-CoV-2 virus, represents a major global health challenge. It is devastating the lives of people around the world. The scientific community, through the application of advanced scientific techniques, can play an important role in helping to relieve some of the suffering. However, for this to be effective, changes are needed in the way scientific research is conducted. Silo mentality in science has slowed down progress, despite greater awareness regarding breaking barriers between different spheres of knowledge. The story of five blind men [Fig. 1] each giving their description about the elephant, on the basis of what they touched, is equally applicable in the way scientific research is carried out. Often it is not possible to get the "full picture" of a particular system due to research being confined within boundaries set by specific techniques or disciplines. The Covid-19 pandemic provides a window of opportunity to break down such barriers and develop better coordination, cooperation and collaboration to achieve scientific breakthroughs, at least in areas where saving human lives is concerned. There is no doubt that one such area is disease diagnosis and the development of drugs and vaccines.

Spectroscopic and imaging techniques can play a pivotal role in different areas of Covid-19 research, including in diagnosis and drug discovery. Unfortunately, as in other areas of science, silo mentality is slowing down progress [Fig. 2]. There is no single spectroscopic or imaging technique that can provide information on all aspects of a particular molecule or system, and each technique has its advantages and disadvantages. It is almost impossible to find a single country, let alone a single laboratory or institute, which has all the necessary expertise in all the different spectroscopic and imaging techniques. Furthermore, there is very little sharing of samples, data and equipments that can help in the standardisation and harmonisation of procedures that can speed up the adoption of techniques for testing, diagnosis and treatment.

Some positive developments have emerged as a result of the Covid-19 pandemic. This includes establishment of research consortia whose goals are to bring together scientists with different expertise to 


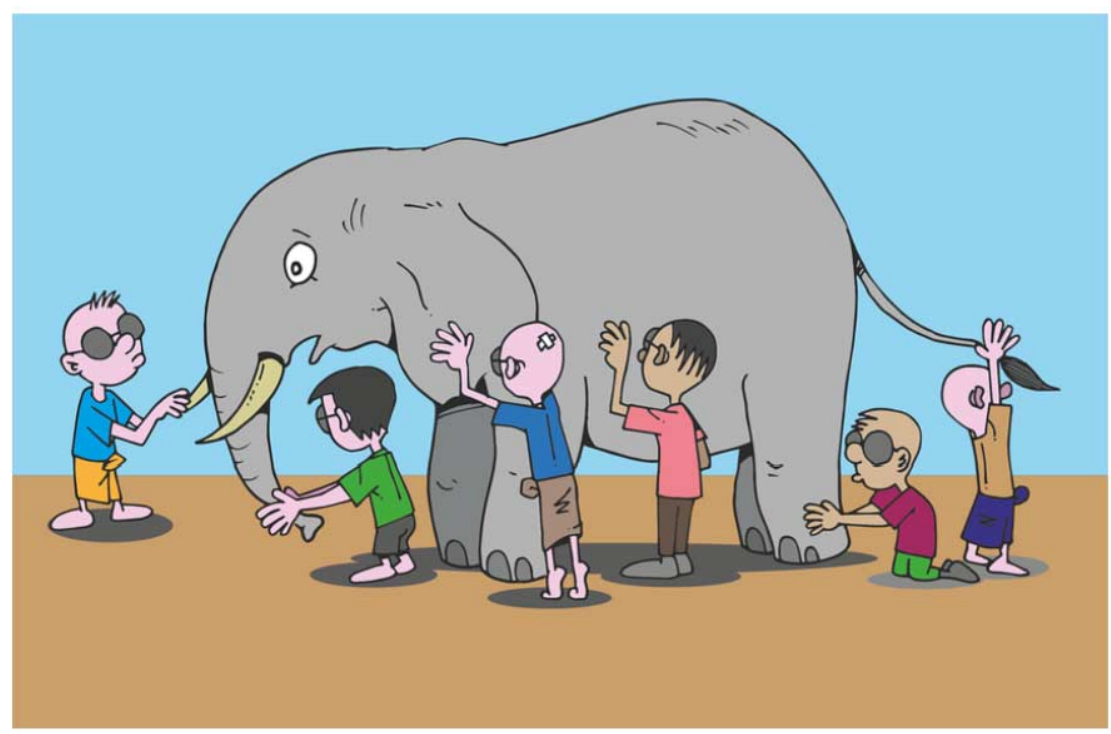

Fig. 1. Five blind men examining an elephant and each providing a different interpretation, based on which part of the animal he touched. This is the problem in much of current scientific research where traditional barriers prevent interaction between different disciplines and techniques leading to an incomplete and partial picture of the system or problem.

advance our understanding of Covid-19. Consortia have been set up covering many different areas of Covid-19 research including genomics [8], immunology [12], diagnostics [9], high-performance computing [4], drug discovery [7], long-term health effects [10] as well as psychological and social impacts of Covid-19 [11]. In the UK, a genomics consortium has identified a new variant of the SARS-Cov-2 virus that spreads more easily between people $[2,3]$

There are several consortia that have been established in the field of spectroscopy. For example, the Covid19-NMR consortium [6] focuses on the application of NMR spectroscopy to determine the structures of RNA and proteins from SARS-CoV-2 with the objective of developing drugs. Another consortium has been established that brings together experts in mass spectrometry to analyse Covid-19 antigens in blood and other bio-fluids [5]. In order to achieve the maximum benefit, the members of this consortium will share samples, processing protocols as well as research data. These are all positive steps and those who have initiated such projects are to be commended for their efforts.

There has not been a better time for research collaboration. It is being catalysed by the common instinct amongst us all to come together to counter an unprecedented threat that does not discriminate between people. Research consortia and collaborative efforts will undoubtedly accelerate our understanding of the virus and will lead to better diagnosis methods as well as development of drugs and vaccines. However, we have to ensure that this "coming together" to solve global health challenges continues beyond the Covid-19 pandemic. We have many global challenges that requires collaboration and pulling together of resources. In my view an opportunity will be missed if we do not break out of the silo mentality and bring together diverse techniques/disciplines, representing different consortia, under an overarching umbrella. Currently, spectroscopic research, like other areas, is restricted by silo mentality (see Fig. 2) that continues to prevail in the scientific community. Spectroscopic and imaging research, especially those addressing global health challenges, needs to be coordinated in such a way that there is greater cooperation and collaboration between researchers using different techniques (see Fig. 3). This will prevent overlap and duplication of effort which is not only financially costly but is also harmful to 

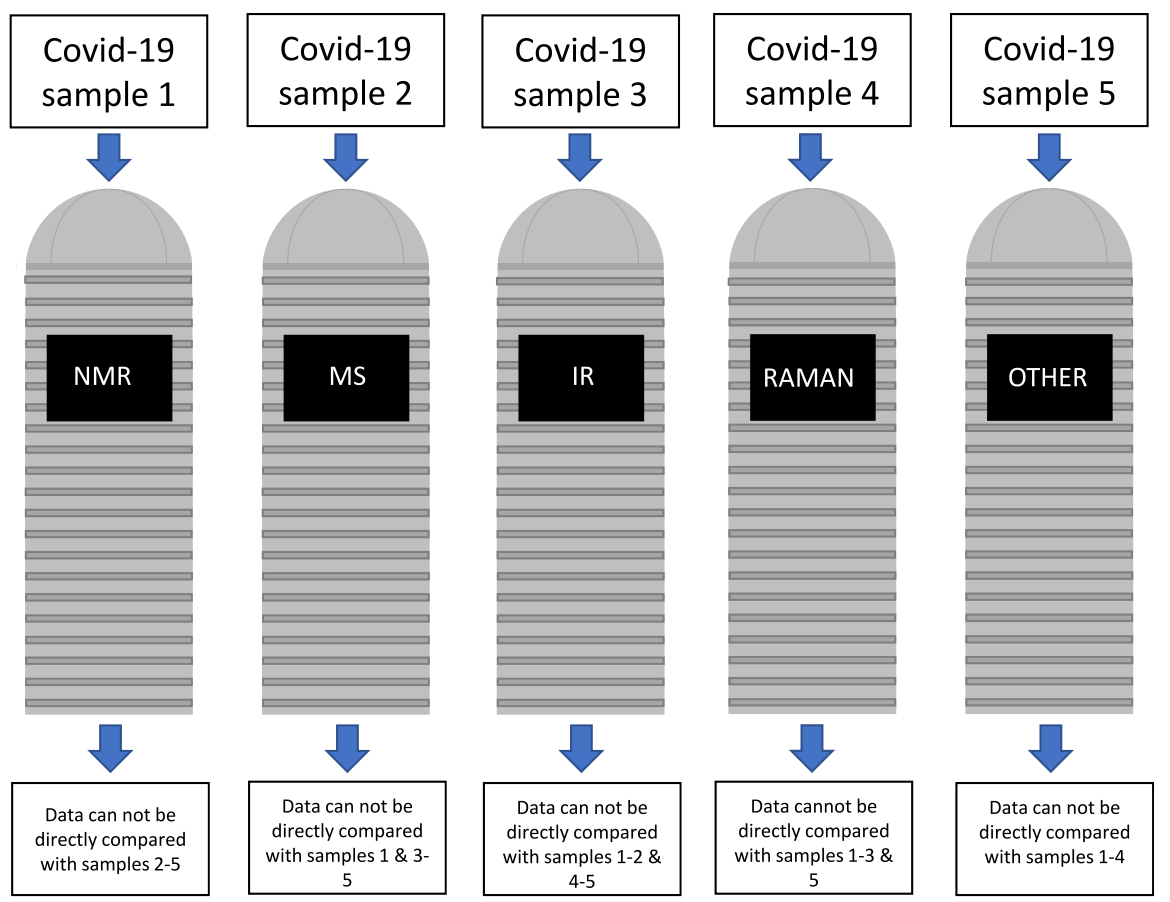

Fig. 2. Even within a specific discipline, research is often carried out in silos where different set of samples (1-5) are analysed using different techniques without sharing of goals, samples and protocols. This prevents comparison between data generated by the different techniques. This reduces the opportunity to complement and supplement the findings from each of the techniques to obtain a better, more comprehensive, "picture" of a particular system or problem. It would be better if the same blood or saliva samples, from Covid-19 patients, were all analysed using each of the spectroscopic techniques and the data and procedures shared to determine intra- and inter-technique variations. This will enable a better understanding of the problem and accelerate the development of spectroscopic techniques as diagnostic tools and their adoption in clinical settings. Spectroscopic techniques given as examples in this diagram are NMR (nuclear magnetic resonance), infrared, Raman and MS (mass spectrometry).

the environment in terms of waste generated and energy consumed. Furthermore, cooperation and collaboration will lead to sharing of ideas and resources that will accelerate progress in the understanding of a disease and developing diagnostic tests and treatments. Just like tonnes of edible food are dumped each year by people, scientists throw away chemicals, consumables, reagents and kits due to a lack of a structure that can identify researchers who may benefit from these materials. In the 21 st century, with advances in information technology, big data and artificial intelligence it should be easy to link researchers and laboratories to prevent such waste of valuable resources whose production and disposal has huge negative impacts on the environment.

Scientists and research funding organisations have a moral obligation to support collaborative research that can accelerate rapid and effective solution to a global pandemic that is adversely affecting the health and livelihood of millions of people around the world. Listed below are some of the steps that can be taken to advance application of spectroscopy and imaging in Covid19 research and indeed for future health challenges (this is also relevant for other techniques and disciplines):

1. Establish an overarching umbrella organisation that will facilitate communication between research consortia that use different spectroscopic and imaging techniques in the study of Covid-19. This will enable sharing of resources and ideas and minimising waste. 


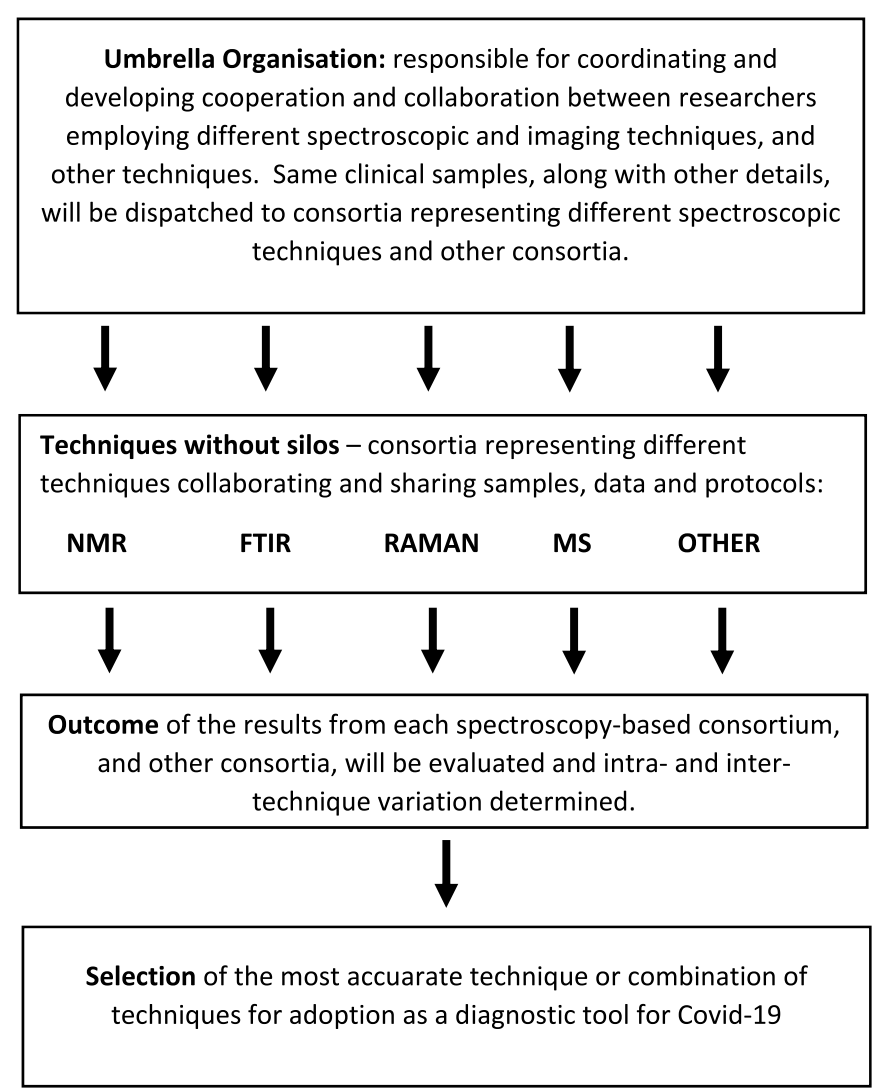

Fig. 3. A diagram showing the key role of the overarching umbrella organisation that will facilitate coordination, cooperation and collaboration between researchers in different consortia, representing different spectroscopic techniques and other techniques. Same set of samples will be analysed by different consortia who will share data, protocols and research goals. This will enable the data from each technique to be directly compared with other techniques so that a more comprehensive picture of the sample can be obtained.

2. A registry of researchers engaged in the application of spectroscopy and imaging techniques can be established for consultation and to identify research priorities and collaborations.

3. Identify and co-operate with experts, from other disciplines, who are needed for making progress in the use of spectroscopic and imaging methods in Covid-19 research. This can include experts in clinical science, mathematical, statistical, computational, engineering, material science, and artificial intelligence techniques.

It would be ideal if these experts could be brought together in a research centre in close proximity to hospitals and medical centres where Covid-19 patients are being treated so that samples and patients will be readily available for research work after appropriate ethical approvals. Alternatively, samples could be sent to different laboratories and to different countries for analysis based on the availability of technique and expertise. If getting everyone together physically in one institution is difficult, alternative strategies could be employed such as having centres focusing on one or more techniques and these centres will co-operate with other centres by sharing knowledge, data and samples. 
The key benefit of such a collaborative effort will be to bring together diverse experts and diverse techniques from around the world for testing, diagnosis and treatment for Covid-19 and thereby saving human lives. It may be a dream, but it is something worth planning considering the greater benefit it will bring to humanity. Currently, there are no global centres that bring together experts in spectroscopic and imaging techniques with the objective to develop testing and diagnosis of diseases. Such a centre will go a long way to accelerate the use of existing spectroscopic and imaging tools to advance the diagnosis of diseases that afflict human beings around the world. Currently, the progress is very slow since the effort is fragmented, lacking coordination, cooperation and collaboration, leading to low complementarity and duplication of efforts.

In this article, I am proposing the formation of an umbrella organisation that acts to facilitate communication, coordination, cooperation and collaboration between researchers engaged in different spectroscopic and imaging techniques. The key role of the overarching umbrella organisation will include the following:

(1) Coordination: In order to reduce overlap and duplication of research, the umbrella organisation will communicate with different spectroscopic research consortia and other relevant consortia regarding research objectives and data collection procedures. Although the different groups may not work together but better coordination of research, facilitated by the umbrella organisation, will help increase efficiency and productivity and reduce redundancy and unnecessary waste of resources.

(2) Cooperation: research consortia using different spectroscopic technique will share their research objectives, plans, protocols and data, thereby creating a better understanding of research goals and reducing intra- and inter-technique friction and mistrust.

(3) Collaboration: research consortia members, representing different spectroscopic techniques, will work together by sharing samples, instruments, protocols and data that will enable harmonisation and standardisation of procedures and the development of the most accurate diagnostic tools.

Details regarding differences between coordination, cooperation and collaboration can be found in a systematic review article by Castañer and Oliveira [1]. The membership of the umbrella organisation should be sufficiently broad to include members of the public (such as patients), academics, clinicians, industry, non-governmental organisations and government. However, this overarching structure should not become a silo itself, but it should connect with other networks and consortia to facilitate seamless interaction and exchange of knowledge between different disciplines of science, health and other areas of knowledge. I hope that one of the positive outcomes of the Covid-19 will be a change in the way scientific research is conducted so that it becomes more holistic, more collaborative and less wasteful.

\section{Conflict of interest}

The author has no conflicts of interest to report.

\section{References}

[1] X. Castañer and N. Oliveira, Collaboration, coordination, and cooperation among organizations: Establishing the distinctive meanings of these terms through a systematic literature review, Journal of Management (2020). doi:10.1177/0149206320901565. 
[2] Covid-19: New coronavirus variant is identified in UK, BMJ 371 (2020), m4857. https://www.bmj.com/content/371/bmj. m4857. Last accessed 21 December, 2020.

[3] https://www.cogconsortium.uk/news_item/update-on-new-sars-cov-2-variant-and-how-cog-uk-tracks-emergingmutations/. Last accessed 21 December, 2020.

[4] https://covid19-hpc-consortium.org/. Last accessed 21 December, 2020.

[5] https://covid19-msc.org/. Last accessed 21 December, 2020.

[6] https://covid19-nmr.de/. Last accessed 21 December, 2020.

[7] https://omicscience.org/apps/covidpgwas/. Last accessed 21 December, 2020.

[8] https://www.cogconsortium.uk/. Last accessed 21 December, 2020.

[9] https://www.crick.ac.uk/research/covid-19/covid19-consortium. Last accessed 21 December, 2020

[10] https://www.phosp.org/. Last accessed 21 December, 2020.

[11] https://www.sheffield.ac.uk/psychology-consortium-covid19. Last accessed 21 December, 2020.

[12] https://www.uk-cic.org/. Last accessed 21 December, 2020. 